\title{
Bilan et modèle d'entrées - sorties du phosphore total dans le Léman
}

\author{
J. M. Jaquet ${ }^{1}$
}

Mots Clés : Limnologie, eutrophisation, phosphore, modèle.

Un bilan d'entrées-sorties du phosphore total pour le Léman entre 1964 et 1983 est présenté. Dans l'ensemble, le lac réalise l'élimination nette de Ptot à un taux moyen de 0,2 an -1 , mais avec de fortes fluctuations, les années à sédimentation nette alternant avec celles à régénération nette. Les conditions météorologiques jouent un rôle capital dans cette alternance.

L'application au Lérnan du modèle simple de Vollenweider et des normes OCDE est discutée. L'approximation du coefficient d'élimination $\phi$ à l'aide du coefficient de renouvellement de l'eau conduit à une forte surestimation de l'élimination.

Pour tenir compte de la variabilité de $\phi$, l'on simule l'évolution du stock selon divers scénarios en suivant une approche stochastique. Il s'avère qu'une variabilité réaliste de $\phi(50 \%)$ peut provoquer des fluctuations interannuelles du stock de olusieurs milliers de tonnes.

Dans les conditions de charge actuelle (1200 $\left.\mathrm{tan}^{-1}\right)$, ou même après une réduction de $400 \mathrm{t}^{-1}$ suite à une interdiction des phosphates dans les détergents, le lac ne pourra pas atteindre l'objectif d'un stock à $2000-3000 \mathrm{t}$.

\section{A budget and input-output model for total phosphorus in Lake Léman.}

Keywords: Limnology, eutrophication, phosphorus, model.

A total phosphorus input-output budget for Lake Léman is presented and discussed for a 20-year period, during which a net elimination predominates at a $\phi$ rate of $0.20 \mathrm{yr}^{-1}$. This coefficient, however, greatly varies from year to year, net sedimentation or net regeneration occurring as a function of meteorological conditions (winter temperature and river discharge).

The application of Vollenweider's model and OECD norms is discussed. It is shown that an approximation of $\phi$ by flushing rate alone would grossly overestimate true elimination.

To allow for $\phi$ variability, the phosphorus stock evolution is stochastically simulated according to several schemes of variation in loading. It is shown that a realistic variability of $\phi(50 \%)$ may induce interannual stock fluctuations of several thousand tons. Under the present loading conditions $\left(1200 \mathrm{t} \mathrm{yr}^{-1}\right)$, and even after a $400 \mathrm{t}$ reduction stemming from a ban on detergent phosphate, the lake will not reach the target stock of 2000-3000 t.

\section{1. - Introduction}

L'eutrophisation du Léman, et plus spécialement le rôle du phosphore dans ce processus, n'ont donné lieu qu'à un nombre restreint de travaux (Haubert et al 1975), Lachavanne 1980). Plus récemment, la Cornmission Internationale pour la Protection du Léman contre la Pollution (CIPEL, 1984) a publiè un rapport de synthèse dont une partie (Chapitre 6.2

1. Unité de Biologie Aquatique, Université de Genève, 18 chemin des Clochettes, 1206 Genève, SuISSE. et Conclusions) représente une première tentative de modélisation du cycle du phosphore dans ce lac.

Il paraît maintenant opportun de dresser un bilan d'entrées-sorties du phosphore total dans le Léman. et d'examiner dans quelle mesure un modèle simple de type Vollenweider (OCDE, 1982) peut contribuer à l'interprétation des données à disposition, à la compréhension du système lémanique et à la simulation de ce dernier dans un but de gestion. 


\section{2. - Base de données et méthodes de calcul}

Les données sur le bilan hydrologique sont tirées du rapport de synthèse de la CIPEL $(1984$, p. 48). Celles qui concernent le phosphore proviennent de la même source (p. 614 et 644), ainsi que de la banque de données et des rapports annuels de la CIPEL. Les taux de sédimentation du phosphore particulaire sont tirées de Jaquet et al. (1983).

Le bilan d'entrées-sorties du Ptotal peut s'exprimer ainsi :

dPJ (Variation annuelle du stock $d \mathbf{u}$ lac) $=\mathbf{L}$ (Apports externes) $+\mathrm{R}$ (Apports internes) - $\mathrm{E}$ (Exports par l'exutoire) - S (Exports par sédimentation).

Le terme dPJ est égal à la différence de stock entre les mois de janvier successifs (Edmondson et Lehman 1981). Si la valeur manque pour ce mois, on l'interpole linéairement entre novembre et février (1964-1969), ou entre septembre et février pour 1967 exceptionnellement.

Les apports externes (CIPEL 1984, p. 644) ont été corrigés à partir des hypothèses suivantes :

- Les apports par les affluents sont considérés comme corrects à l'exception de 1977, année pour laquelle on a appliqué la relation $\mathrm{L}=\mathrm{Q} \times \overline{\mathrm{Pj}}, \overline{\mathrm{Pj}}$ étant la valeur moyenne entre 1976 et $1978(122 \mathrm{mg}$ $\mathbf{m}^{-3}$, voir Tableaux I et II).

- Les apports par précipitation sur le lac $(60 \mathrm{t}$ $a^{-1}$ en moyenne) ont été rajoutés de 1964 à 1971.

- Les apports par les STEP se déversant directement dans le lac sont sous-estimés : suivant la sug. gestion d'Ammann (1984), nous avons multiplié par un facteur de trois les chiffres de la CIPEL, et estimé à $600 \mathrm{t}$ ces apports pour les années $1964-1966$.

- Les apports par ruissellement direct n'étant pas mesurés par la CIPEL, les valeurs de la charge externe (L, Tableau II) représentent un minimum. Il en résulte donc probablement une surestimation de la charge interne.

- Les exportations à l'émissaire sont égales aux valeurs de la CIPEL (1984, p. 644). A noter que tous les chiffres du Tableau II ont été arrondis.

- Les apports internes et les exportations par sédimentation (à l'exception de 1980-81 pour ces derniers, Jaquet et al, 1983) n'ont pas été mesurés dans le Léman. Ils ont donc été estimés à l'aide de l'équation du bilan (voir plus loin).
Tableau I. - Liste des paramètres et variables (Movennes on totaux annuels ; $\mathrm{P}$ comme Ptotal)

En première approximation, et afin de simplifier l'équation du bilan, il est possible de se baser sur un certain nombre d'hypothèses (OCDE 1982, Gaechter et al 1983) :

Tout d'abord, les exportations peuvent être considérées comme dépendant linéairement de la concentration moyenne en Ptot du lac, ce qui conduit aux expressions suivantes (voir Tableau I pour abréviations et OCDE 1982 pour définitions).

$\mathrm{E}=[\mathrm{P}] \quad \vee \quad \beta \quad \rho$

où $\beta$ rend compte du fait que la concentration de l'exutoire est en général plus basse que celle du lac. Pour les exportations par sédimentation.

$$
\mathbf{S}=[\mathrm{P}] \quad \mathrm{V} \quad \boldsymbol{\theta}
$$

Du fait que les exportations par sédimentation n'ont été que rarement mesurées, la relation (3) ne 
Tableau II. - Données du bilan et paramètres dérivés.

\begin{tabular}{|c|c|c|c|c|c|c|c|c|c|c|c|c|c|c|c|c|c|c|c|c|}
\hline & 0 & as & $I_{w}$ & $*$ & fn & $L$ & A L & $[D]]$ & $\cos 1$ & E & {$[D] 6$} & $[P]]=$ & Place & Eet & 618 & pni & n 1 & $T_{p}$ & $a_{1}$ & se \\
\hline$A N$ & $\begin{array}{c}\text { Mo. } \\
\text { an }\end{array}$ & m/an & en & $a n-1$ & $a n-1 / 2$ & tran & $\underset{m}{\sin }$ & $\operatorname{mog}_{\mathrm{m}}$ & $m$ & tran & $m$ & $m_{m}$ & c & & & & & ans & 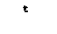 & t \\
\hline 1964 & bo3rs & 10.4 & 14.8 & 0.07 & 0.21 & 1040 & 1.8 & 172 & 36 & 260 & 43 & 62 & 5510 & o. 70 & 0.65 & 0.70 & 0,36 & $\Rightarrow .3$ & -2000 & -3500 \\
\hline 1965 & 7480 & 1 ह. 9 & 11.9 & 0.00 & 0.22 & $1 \geq \theta$ & $e . \varepsilon$ & 171 & 38 & 250 & 3.3 & 59 & 5240 & 0.57 & -0.16 & -0.11 & c. 34 & 4. 3 & 1050 & $B \geq 0$ \\
\hline 1766 & B59 & 14.0 & 10.4 & a. 10 & 0,24 & 1350 & 3.4 & $23 E$ & 35 & 180 & $E 1$ & 44 & 3940 & 0.48 & 1.02 & 1.06 & 0.19 & 2.0 & -2200 & -4010 \\
\hline 1967 & 7940 & 13.6 & $1 \mathrm{~b} .2$ & 0.09 & 0.23 & 1400 & 2.5 & 186 & 43 & 190 & 24 & 28 & 2470 & 0.85 & 0,53 & 0.61 & 0.15 & 1.7 & -30 & -1320 \\
\hline 1968 & Boso & 13.9 & 11.0 & 0.09 & 0.83 & 1040 & $1 . \theta$ & 129 & 30 & esto & 31 & 43 & 3660 & 0.72 & -0.20 & -0.13 & 0.33 & 3.7 & 1550 & 760 \\
\hline 1969 & $72 \%$ & 12.5 & 12.2 & 0.06 & 0.82 & 960 & 1.6 & $13 \varepsilon$ & २9 & 280 & 38 & 50 & 4440 & 0.77 & -1.08 & -1.02 & 0.30 & 4.6 & 5400 & 4000 \\
\hline 1970 & 9070 & 15.6 & $3, \mathrm{~B}$ & 0.10 & 0.24 & 2240 & 3.8 & 247 & 50 & 400 & 44 & 100 & 9590 & 0.41 & 0.55 & 0.59 & 0.44 & 4.3 & -3390 & -5230 \\
\hline 1971 & 6320 & 10.9 & $1 \bullet .1$ & 0.07 & 0.21 & 1650 & 2. 8 & 261 & 55 & 280 & 44 & 67 & 5980 & 0.66 & 0.31 & 0.36 & o. 26 & 3.6 & -490 & $-1 A 60$ \\
\hline 1972 & 6140 & 10.5 & 14.5 & 0.07 & 0.21 & 1240 & e. 1 & $20 \hat{2}$ & 42 & 250 & 41 & 73 & 6400 & 0.57 & -0.15 & -0.12 & 0.36 & 5.2 & 1980 & 990 \\
\hline 1973 & 7290 & 12.5 & เ2. 2 & 0.08 & 0.32 & 1230 & 2.1 & 169 & 38 & 380 & 53 & 93 & 7370 & 0.63 & 0.36 & 9.31 & 0.49 & 6.8 & -1090 & -1949 \\
\hline 1974 & 7060 & 12.1 & 12.6 & 0.08 & $0,2 z$ & 1040 & 1.8 & 147 & 32 & 440 & 62 & 80 & 7070 & $0.7 \mathrm{~B}$ & -0.02 & 0.04 & 0.54 & 6.6 & 740 & 140 \\
\hline 1975 & $6 \bar{\varepsilon} 3 C_{1}$ & 34.1 & 10.8 & 0.09 & 0.23 & 1440 & 2.5 & 175 & 41 & 520 & 63 & es & 7570 & 0.74 & -0.08 & -0.01 & 0.49 & 5.3 & 1500 & 580 \\
\hline 1976 & $\mathbf{5 9 3 0}$ & 10.2 & 15.0 & 0.07 & 0.21 & 950 & 1.6 & 160 & 33 & 290 & 49 & 92 & 8160 & 0.53 & 0.09 & 0.13 & 0.57 & B. 6 & -100 & -760 \\
\hline 1977 & 8970 & 15.4 & 9.9 & 0.10 & 0.24 & 1420 & 2. 4 & 158 & 39 & 450 & 50 & 91 & 8070 & 0.55 & 0.23 & 0.28 & 0.57 & 5. 7 & -870 & -2040 \\
\hline 1978 & 8870 & 15.2 & 10.0 & 0.10 & 0.24 & 1380 & 2.4 & 156 & 37 & 4.30 & 40 & eA & 7850 & 0.55 & 0.11 & 0.16 & 0.57 & 5,7 & 120 & -830 \\
\hline 1979 & 8450 & 14.5 & 10.5 & 0.09 & 0.24 & 1390 & 2,4 & 164 & 39 & 630 & 75 & 30 & 8020 & 0.83 & 0.11 & 0.19 & 0.55 & 5.8 & -120 & -880 \\
\hline 1980 & 9200 & 15.8 & 9.7 & 0.10 & 0.24 & 1390 & 2.4 & 151 & 37 & 630 & 6.8 & 84 & 7450 & 0.82 & 0.210 & $0.2 \mathrm{~A}$ & 0.56 & 5.4 & -720 & -1480 \\
\hline 1581 & 9780 & 16.8 & 9.1 & 0.21 & 0.25 & 1280 & e. 2 & 131 & 33 & 630 & 64 & 82 & 7250 & 0.79 & 0.14 & 0.22 & 0.63 & 5.7 & -340 & -990 \\
\hline 1982 & 9800 & 16.8 & 9.1 & 0.11 & 0.25 & 1390 & 2.4 & 142 & 35 & 530 & 60 & 77 & 6050 & 0.78 & 0,38 & 0.46 & 0.54 & 4.9 & -1770 & -2570 \\
\hline 2903 & esoc & 14.70 & 9.60 & 0.10 & 0.24 & 2036 & 1. & 122 & 30 & 530 & 52 & 75 & 6640 & 0.83 & -0.04 & 0.04 & 0.62 & 5.9 & 800 & 294 \\
\hline mox. & 351 & & 11.4 & .09 & 0.23 & 1343 & 2.3 & 170 & 39 & 393 & 49 & 73 & 6487 & 0.68 & 0.14 & 0.20 & 0.45 & 5.0 & 5 & -945 \\
\hline$v(x)$ & 15 & 13 & $\begin{array}{r}1.9 \\
16\end{array}$ & 15 & 0.01 & $\begin{array}{r}321 \\
24\end{array}$ & $\begin{array}{r}8.6 \\
24\end{array}$ & $\begin{array}{l}38 \\
\text { be }\end{array}$ & 21 & $\begin{array}{r}130 \\
38\end{array}$ & 30 & 27 & $\begin{array}{r}176 \\
27\end{array}$ & $\begin{array}{r}0.13 \\
19\end{array}$ & $\begin{array}{r}0.1 \\
2 B 8\end{array}$ & 201 & & 30 & 38308 & - rese \\
\hline $\mathbf{N}$ & 20 & 2o & 20 & 20 & 20 & 80 & 20 & 20 & 20 & 20 & 20 & 20 & 20 & 20 & 20 & 20 & 20 & 20 & 20 & 20 \\
\hline
\end{tabular}

peut pas encore ètre vérifiée. Dans certains cas, $\mathbf{S}$ dépendrait plutôt de [P]j (lake Washington, Edmondson \& Lehman 1981).

Dans le modèle de Vollenweider (OCDE 1982), les apports internes sont censes être nuls. Leur expression est encore plus difficile que celle de la sédimentation, les mécanismes de la régénération n'étant pas encore connus de manière quantitative.

Si l'on suppose (Premazzi \& Rossi 1984) que l'accroissement de la concentration en phosphore près de l'interface eau-sédiment indique l'existence d'un flux vertical par régénération, on peut en déduire une certaine interdépendance entre ce flux et $[P]$ (concentration moyenne dont la valeur, dans le Léman, est très influencée par celle de l'hypolimnion). On aurait donc approximativement

$$
\mathrm{R}=[\mathrm{P}] \quad \mathrm{V} \boldsymbol{\alpha}
$$

L'approximation de l'équation du bilan, en termes de stock, peut s'écrire :

$$
\begin{aligned}
& \mathrm{V} d[\mathrm{P}] / \mathrm{dt}=[\mathrm{P}] \mathrm{e} \mathrm{V}+[\mathrm{P}] \mathrm{V} \alpha-[\mathrm{P}] \beta \varrho \mathrm{V}-[\mathrm{P}] \\
& \theta \vee(5)
\end{aligned}
$$

$\begin{array}{llll}\text { Apports } & \text { Apports } & \text { Exports } & \text { Exports } \\ \text { externes } & \text { internes } & \text { exutoire } & \text { sédimen. }\end{array}$

En l'absence d'estimations annuelles de $\mathrm{S}$ et de $R$, il est nécessaire de regrouper ces deux termes sous le nom de sédimentation-régénération nette $\mathrm{SR}$.

$$
\mathrm{Vd}[\mathrm{P}] \mathrm{dt}=[\mathrm{P}] \varrho \mathrm{V}-[\mathrm{P}] \beta \varrho \mathrm{V}-[\mathrm{P}] \mathrm{V} \sigma
$$

où $\sigma=\theta-\alpha$

Le coefficient $\sigma$, suivant son signe, exprime donc les effets antagonistes de la sédimentation et de la régénération. En l'absence de sources internes $(\alpha=$ $0)$, il est égal à $\theta$.

La solution de (6) est :

$$
[\mathbf{P}]=[\mathbf{P}]^{0} \mathrm{e}^{-\phi_{\mathrm{t}}}+[\mathbf{P}] \mathbf{j} \pi-[\mathbf{P}] \mathbf{j} \pi \mathrm{e}^{-\phi_{\mathrm{t}}}
$$

où $[\mathrm{P}]^{0}$ est la concentration du lac à $\mathrm{t}=0, \pi=$ $1 /(\beta+\sigma \tau)$ et $\phi=\beta \mathrm{Q}+\sigma$ (Tableau I) 
A l'équilibre $(\mathrm{d}[\mathrm{P}] \mathrm{dt}=0$ ou $\mathrm{t} x),(6)$ et $(7)$ se simplifient en

$$
[\mathrm{v}]^{\infty}=[\mathbf{P}] \boldsymbol{j} \pi
$$

On comparera plus loin cette expression avec la relation statistique calculée à partir des diagrammes de l'OCDE (1982)

$$
\begin{aligned}
& {[\mathrm{P}]=1.55([\mathrm{P}] \mathrm{i})^{0.82}} \\
& \text { où }[\mathrm{P}] \mathrm{i}=[\mathrm{P}] \mathrm{j} \mathrm{f}_{\mathrm{h}} \\
& \text { et } \mathrm{f}_{\mathrm{h}}=1 /(1+\sqrt{\mathrm{T}})
\end{aligned}
$$

En termes de stock (tonnes), les équivalents de (7) et (8) sont :

$$
\begin{aligned}
& \mathrm{P}=\mathrm{P}^{0} \mathrm{e}^{-\phi_{\mathrm{t}}}+\mathrm{L} \phi-\mathrm{L} \phi \mathrm{e}-\phi_{\mathrm{t}} \\
& \text { et } \\
& \mathrm{P}^{\infty}=\mathrm{L} 1 / \phi \quad \text { pour } \phi>0
\end{aligned}
$$

Enfin, le temps nécessaire (années) pour atteindre une certaine fraction $\mathrm{f}$ de $\mathrm{P}^{\infty} \mathrm{s}^{\prime}$ exprime par :

$\mathrm{t}_{\mathrm{f}}=\mathrm{F}_{\mathrm{f}} 1 / \phi$

avec $F_{0.9}=2.3, F_{0.95}=3.0$ et $F_{0.99}=4.6$.

\section{3. - Bilan d'entrées-sorties}

Ce bilan est exprimé en chiffres dans le Tableau II et graphiquement sur la fig. 1. On se reportera à Jaquet \& Snodgrass (1984) pour une discussion détaillée de ces résultats. Rappelons seulement que les valeurs de SR avant 1970 sont sujettes à caution dans leur magnitude, à cause de l'imprécision dans l'estimation des apports externes.

Avant 1975, on remarque la succession chaotique d'années à régénération nette $(1965,1968,1969$. 1972) suivies d'épisodes à sédimentation nette (1964, $1966,1970,1973)$. Une comparaison avec le graphique du bas de la fig. 1 suggère que la régénération à l'interface eau-sédiment suit l'établissement de gradients négatifs de $[0]_{2}$ à $300 \mathrm{~m}$. Toutefois, la régénération maximale ne coincide pas toujours avec les minima de l'oxygène (1978), comme si le stock échangeable de phosphore du sédiment était épuisé (Jaquet 1978).

On peut attribuer les épisodes de forte sédimentation à l'abondance relative de phosphore particulaire, soit apporté par les affluents les années à fort débit (1966, 1970, 1982), soit d'origine biologique (seston).

Il est possible de vérifier, pour l'année 1980, l'estimation de la sédimentation obtenue par bilan (1480 tonnes, Tableau II) : Jaquet et al. (1983) ont mesuré,

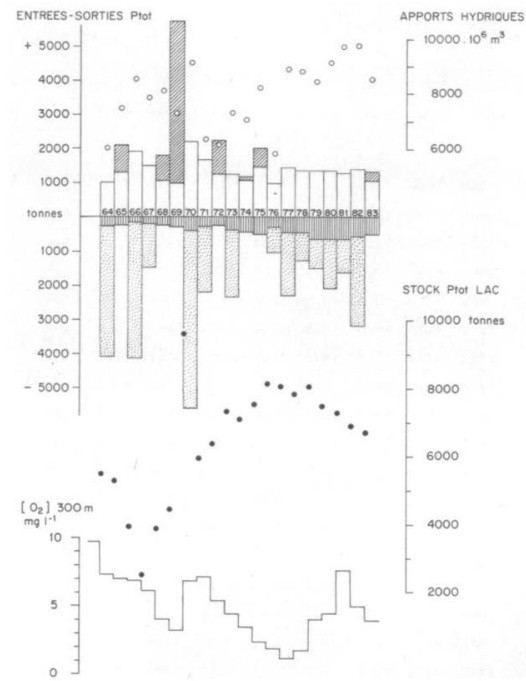

Fig. 1. Expression graphique du bilan et évolution du stock de $P$ total et de la concentration en oxygène à $300 \mathrm{~m}$.

à l'aide de trappes et en deux stations du lac, le taux de sédimentation des formes particulaires du phosphore. A raison d'environ 400 t de $\mathrm{P}$ organique et de $800 \mathrm{t}$ de $\mathbf{P}$ minéral, on arrive à une valeur moyenne de $1200 \mathrm{t}$ de $\mathrm{P}$ total.

Pour cette année-là, les deux types d'estimation donnent donc des résultats du même ordre de grandeur. Ils sont représentatifs des quantités de $\mathbf{P}$ total sédimentées dans les années 80 , caractérisées par des apports hydriques supérieurs à la moyenne (Table II, Q).

\section{4. - Examen critique de quelques hypothèses du modèle.}

\section{1. - Linéarité}

Une des principales présuppositions à la base du modèle exprimé par les équations 7 et 12 est que les coefficients $\pi$ et $\phi$ sont constants et ne dépendent pas de $\mathrm{P}$ (hypothèse de linéarité, Gaechter et al., 1983).

Or, on constate dans le Tableau II que de 1964 à 1983 , le coefficient de variation de $\pi$ est de $31 \%$ et celui de $\phi$ de $201 \%$. Cette instabilité provient de la 
variabilité de e (15\%), de $\beta$ (19\%) et surtout de $\sigma$ (près de $300 \%$ ). Il s'agit donc d'examiner (a) si ces coefficients sont des fonctions de $P$, auquel cas un modèle linéaire ne serait pas applicable au Léman et (b), s'ils sont corrélés entre eux ou avec une ou plusieurs variables externes.

\subsubsection{Le coefficient d"élimination hydraulique e}

Ce coefficient, égal à l'inverse du temps de séjour théorique de l'eau, est mesuré avec une bonne précision (CIPEL 1983, p. 48). Sa variabilité dépend donc uniquement des facteurs climatiques tels que pluie, neige et fonte estivale. Il n'est d'autre part corrélé significativement ni avec $\beta, \sigma$ ou $P$.

\subsubsection{Le coefficient de mélange $\beta$}

Absent dans le modèle originel de Vollenweider (1968), ce coefficient a été introduit plus tard (Sonzogni et al. 1976) pour tenir compte de la vidange d'eau épilimnétique appauvrie en phosphore pendant la période de stratification.

Dans le Léman, $\beta$ varie entre 0,4 et 0,8 , avec une moyenne de $0,68 \pm 0,26$ (Tableau II et fig. 2). Ainsi, pour une concentration moyenne de l'eau du lac voisine de $85 \mathrm{mg} \mathrm{m}^{-3}$, la concentration moyenne à l'exutoire peut varier de 50 (1976.78) à $75 \mathrm{mg} \mathrm{m}^{-3}$ (1979). Un examen de la fig. 2 et des courbes de température à 150 et $300 \mathrm{~m}$ (CIPEL 1984 p. 560) indique que les hautes valeurs de $\beta$ correspondent à des années ayant débuté par un bon mélange des eaux, donc caractérisées par un hiver froid et venteux.

Dans l'équation exprimant les exportations (2), $\beta$ est un facteur diminutif. Multipliant $\varrho$, il revient à décroitre l'efficacité de la vidange, dont la valeur moyenne descend à $0,061 \pm 0,032 \mathrm{an}^{-1}$. Ce coefficient n'est pas corrélé significativement avec $\varrho, \sigma$ ou $P$.

\subsubsection{Le coefficient de sédimentation-régénération $\sigma$}

Parmi les coefficients d'élimination examinée, $\sigma$ est certainement le moins fiable. En effet, il représente le résultat de deux processus antagonistes, la sédimentation et la régénération, lesquels ne sont pas mesurés, mais calculés par différence dans le bilan. Or, l'on sait que la précision des mesures d'apports laisse à désirer, en tous cas jusqu'en 1975. Il est donc probable qu'une partie de la forte variabilité de $\sigma$ (Tableau II) soit due à ces imprécisions (le coefficient de variation de $\sigma$ passe de $400 \%$ à $80 \%$ après 1976).

Néanmoins I'alternance, déjà mentionnée, d'années à sédimentation nette et à régénération nette

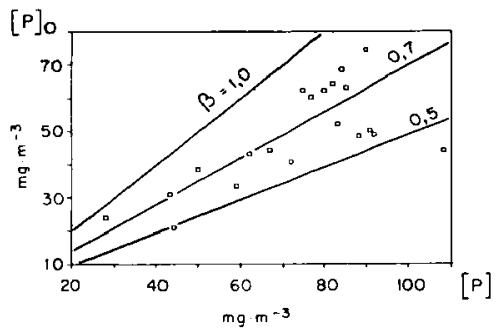

Fig. 2 : Relation entre la concentration en Ptot du lac et de l'exutoire et variation de $\beta$.

peut être acceptée, quand bien même les tonnages mis en cuvre sont certainement plus faibles que ceux qui apparaissent dans le Tableau II et la fig. 1. Le coefficient $\sigma$ est donc intrinsèquement variable.

Malheureusement, son imprécision va masquer les relations qu'il pourrait avoir avec les aut res paramètres : une analyse de corrélation linéaire pas à pas montre que $\sigma$ n'est lié significativement $(p=0.01)$ qu'à $L(r=0,61)$. Ce fait est à rapprocher des résultats d'Edmondson et Lehman (1981) dans le Lake Washington, où l'élimination par sédimentation est fonction des apports, et non de la concentration annuelle moyenne de $\mathbf{P}$ total dans l'eau du lac.

Conceptuellement, l'on conçoit que la quantité de phosphore sédimenté dépende des apports par le biais de la phase particulaire, qui peut atteindre, dans le Rhône, les $70 \%$ du total (Jaquet et Snodgrass 1984, fig. 4). En outre, une forte production primaire pourrait, elle aussi, accélérer la sédimentation sous forme de $\mathrm{P}$ organique.

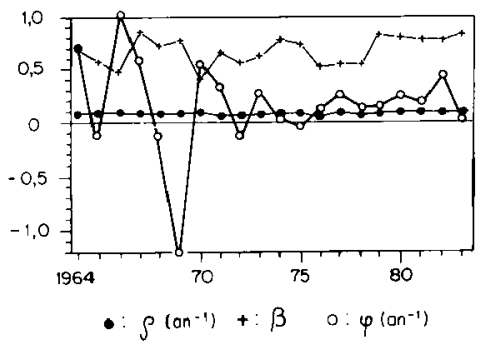

Fig. 4: Evolution chionologique de $e, \beta$ el $\phi$ 
D'un autre côté, l'absence de mélanges hivernaux plusieurs années de suite (1971 à 1978, voir CIPEL 1984, p. 560) conduit à l'anoxie de l'interface eausédiment et à la régénération de $\mathrm{P}$ réactif soluble, donc à la présence de sources internes: $\alpha$ prend alors le pas sur $\theta$ et $\boldsymbol{\sigma}$ diminue. On voit par ces exemples que la variation de $\sigma$ répond à des causes multiples et complexes, climatiques autant que trophiques.

\subsubsection{Le coefficient d'élimination globale $\phi$}

Ce coefficient regroupe les effets de tous les processus d'élimination ou d'apports internes dans le système lacustre. D'une année à l'autre, $\phi$ peut varier fortement : lorsqu'il est négatif, il indique une régénération nette à partir du sédiment. Dans ce cas, il découle de (12) que pour des apports externes constants, $P$ augmente.

Dans la plupart des cas toutefois, $\phi>0$ et sur la période considérée, le Léman réalise une élimination nette de son phosphore. Au vu des valeurs res. pectives de $\beta$, $\varrho$ et $\sigma$, et des résultats de la fig. 2 , il est clair que cette élimination se fait aux $2 / 3$ par sédimentation (Tableau II).

Au terme de cet examen des coefficients de l'équation du bilan, on peut faire les remarques suivantes :

1. Il n'y a pas évidence d'une corrélation entre $P$ d'une part et $\phi, \beta$ et $\sigma$ de l'autre. Le modèle exprimé par (6) peut être considéré comme linéaire, si l'on prend pour valeurs des coefficients leur moyenne pluriannuelle. Si l'on admet en revanche que ces coefficients varient au cours du temps de manière significative. (7) n'est pas la solution de (6). On en verra plus loin les implications au niveau de la simulation.

2. Les coefficients $\phi, \beta$ et $\sigma$ ne sont pas corrélés entre eux. Ils représentent de manière simplifiée les processus de vidange hydraulique, de mélange hivernal et de sédimentation-régénération.

3. La variabilité de $\phi$ et $\beta$ (15-20\%) est intrinsèque, et dépend des conditions météorologiques. En revanche, il convient de défalquer de la forte variabilité de $\sigma$ la part due au calcul par différence. Néanmoins, elle demeure forte, ce qui rendrait peu réaliste une simulation avec $\sigma$ constant.

\section{2. - Equilibre de $P$ avec $L$.}

\subsubsection{Cas theoriques}

Par rapport à (12), l'on peut envisager deux cas de non-équilibre :

a) L étant constant, $\mathrm{dP} / \mathrm{dt}+0$ tant que $\mathrm{t} \leqslant 3 \tau(14)$. moment ou $P / P^{X}=0,95$. Dans le Léman, pour un coefficient d'élimination $\phi$ moyen $\left(0,15 \mathrm{an}^{-1}\right.$, ou $\tau_{\mathrm{p}}=6$ ans), cette période de non-équilibre est donc d'environ 18 ans. En d'autres termes, le lac mettra une vingtaine d'années pour atteindre un stock de phosphore stable correspondant à un certain niveau des apports. Pendant ce laps de temps, $\mathbf{P}$ mesuré sera inférieur à $P$ en " phase de croissance " (anatrophisation) et supérieur en " phase de décroissance * (catatrophisation).

b) Si L nest pas constant, la situation est plus complexe. Dillon (1974) et Sonzogni et al. (1976) ont examiné divers types de variation de $\mathbf{L}$. Le cas le plus simple est celui d'un changement brusque d'une valeur constante à une autre. La fréquence temporelle de ces changements permet ou non l'établissement de l'équilibre.

Dans le cas d'un accroissement régulier de $L$, par exemple $\mathrm{L}_{\mathrm{i}}=\mathrm{L}_{\mathrm{i}-1}+\mathrm{dL}$ ou $\mathrm{L}=\mathrm{A}$ ! ( $\left(\mathrm{A}\right.$ en tonne $a^{-2}$ ), on peut définir le coefficient de variation relative des apports

$$
\begin{aligned}
& \eta=A / L \text { (années }-1 \text { ) (Gaechter et al. 1983) (15) } \\
& \mathrm{F}=\eta / \phi \quad \text { (16) }
\end{aligned}
$$
et

Ces rapports permettent une comparaison entre le taux de variation des apports et l'élimination. Sonzogni et al. (1976) ont démontré que le tonnage n'atteint jamais une valeur d'équilibre, mais est égal à :

$$
P=A t / \phi-A / \phi^{2}\left(t-e^{-\phi_{t}}\right)
$$

Le premier terme représentant la valeur à l'équilibre en supposant $\mathrm{L}=\mathrm{ct}$ et le deuxième le retard (lag) qui tend vers $\mathrm{A} / \phi^{2}$ quant $\mathrm{t}>3 \tau$.

\subsubsection{Périodes d'équilibre-déséquilibre}

Pratiquement, l'on peut considérer le lac et ses apports comme étant en équilibre si $\mathrm{P} / \mathrm{P}^{x}$ est supérieur à un certain seuil, fixé ici arbitrairement à 0,9 . On peut donc déterminer les valeurs maximales de $\eta$ à ne pas dépasser, et ce en fonction de $\phi$ fig. 3). Pour une valeur moyenne de ce dernier paramètre $\left(0,20 \mathrm{an}^{-1}\right), \eta=0,02 \mathrm{an}^{-1}$. Plus généralement, on peut démontrer que pour respecter $P / P \geqslant 0,9$, il faut que $F \leqslant 0,1$. Dans le cas du Léman, $(\phi=0,20$ 


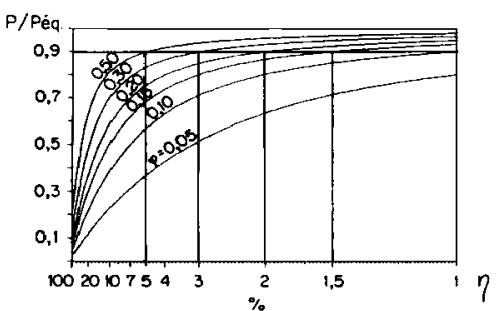

Fig. 3 : Détermination des valeurs-limites de $\eta$ en fonction de $P / P^{x}$.

et $\mathbf{L}=1200$ ), on obtient $\eta=0,02$ et $\mathbf{A}^{\max }=25 \mathrm{t}$ $a^{-2}$. Si l'augmentation des apports dépassait cette valeur, le décalage entre stock mesuré et stock à l'équilibre tendrait vers $600 \mathrm{t}$. Plus A est élevé (pour $\phi \mathrm{ct}$ ). plus le décalage entre stock réel et stock à l'équilibre sera grand.

Sur la base des données de la CIPEL (1984 p. 121 sq : fig. 3, p. 554), et en gardant à l'esprit l'incertitude attachée aux mesures d'avant 1970 , on peut diviser en trois phases l'évolution des apports au Léman (Tableau III) :

Tableau III. - Périodes d'équilibre-déséquilibre du stock de Plot en fonction des apporis.

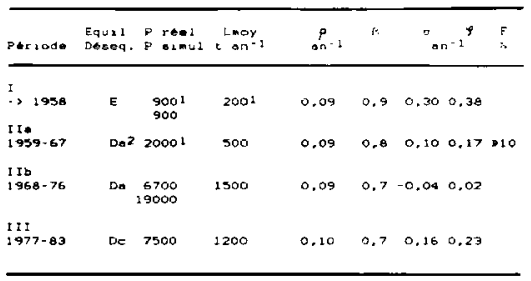

1 Eetimetion

2 Da: destquilibe en anatrophie: DC: destquilibre en cototrophie

1. Du début du siècle jusque vers 1958 , la concentration de Ptot du lac est stable autour de $10 \mathrm{mg}$ $\mathrm{m}^{-3}(900 \mathrm{t})$. On peut donc admettre que le système est à l'équilibre et calculer des apports d'environ 200 $t$ à l'aide de (13) et des valeurs suivantes des coefficients : $\phi=0,09 \mathrm{an}^{-1}, \beta=0,9$ (valeur plus élevée que la moyenne 1960-1980, à cause de l'absence pro. bable de sources internes, donc de forts gradients de concentration hypo-épilimnion), $\sigma=0,30$ an -1 (valeurs moyenne pour années sans régénération, Tableau (I); donc $\phi=0.38 \mathrm{an}^{-1}$.

II. De 1958 à 1976 , il existe de fortes variations des apports d'une année à l'autre, dues principalement à l'apparition de sources internes. En gros, on peut admettre une augmentation jusqu'en 1969-70, suivie d'une décroissance irrégulière. $F$ dépasse souvent le seuil des $10 \%$, mais la variation des apports est erratique, et change de signe tous les 1-3 ans. On doit dons admettre que le lac n'est pas à l'équilibre (fig. 1).

III. Dès 1977, les apports répertoriés deviennent à peu près constants $\left(1200 \mathrm{t} \mathrm{an}^{-1}\right)$, probablement parce que les mesures sont entachées d'une erreur moindre, mais surtout parce que la régénération cesse presque totalement. $\eta$ est donc faible. Néanmoins, on peut voir sur la fig. 1 que le stock de Ptot décroît dès 1976-77, suite à la diminution des apports moyens entre les périodes II et III. Le système est donc en phase catatrophique, le tonnage observé étant plus grand que le tonnage à l'équilibre.

Dans les grandes lignes, on peut donc considérer l'évolution des apports comme une lente phase de croissance jusque dans les années 70 , suivie d'unephase de constance. A cette tendance se superposent des variations parfois très fortes, dues (a) aux erreurs de mesures, (b) aux facteurs météorologiques (agissant sur l'érosion et la sédimentation) et (c) aux sources internes, elles-mêmes dépendant en partie du climat. Ces " catastrophes " rendent difficile l'application stricte des raisonnements proposés par Dillon (1974), Vollenweider (1968) et Sonzogni et al. (1976).

Le fait que le Léman ne soit plus en équilibre avec ses apports depuis bientôt 20 ans doit donc être considéré dans toute tentative d'application du modèle OCDE (1982).

\section{3. - Application des normes OCDE}

Dans le rapport de synthèse de la CIPEL (1984, p. 569), le Léman a été placé au sein du référentiel [P] - [P]i. Ce dernier terme représente la concentration moyenne des apports corrigés par le flux hydraulique (facteur $f_{h}$, relation (11). Ce facteur entre dans l'expression (OCDE 1982, p. 119)

$$
[\mathbf{P}]=[\mathrm{P}] \mathrm{j} /(1+\sqrt{\mathrm{T}}) \text { (18) }
$$


$f_{h}$ est donc l'expression simplifiée de $\pi$ (Tableau I). Pour le Léman, la norme pluriannuelle de $\mathrm{f}_{h}$ vaut 0,23 et celle de $\pi, 0,45$. L'expression statistique conduit donc à une sous-estimation considérable de $[\mathbf{P}]$, due à une surestimation du coefficient d'élimination.

Un second point doit être relevé dans l'interprétation des diagrammes OCDE. On a vu plus haut que le stock n'était que rarement en équilibre avec les apoorts. Ainsi, en 1980, [P] est supérieur à [P] ${ }^{\Upsilon}$ (phase catat rophe), ce qui explique la position excentriquc du Léman sur le diagramme de la CIPEL (1984, fig. 12. p. 569 ).

En outre, le problème de la charge interne se pose aussi dans le contexte de ces diagrammes. En 1969 par exemple (Tableau II, fig. 1), les apports par le sédiment ont été significatifs (plus de $1000 \mathrm{t}$ ?). Il faudrait donc théoriquement les rajouter à la valeur de [P]i, ce qui déplacerait, sur le diagramme précité, le point 1969 vers la droite, et sous la ligne de tendance moyenne. Cette position serait mieux en accord avec le fait que, cette année-là, le lac était en phase anatrophe.

Enfin, l'utilisation de $\mathrm{f}_{\mathrm{h}}$ ou de $\pi$ a aussi des conséquences importantes sur l'estimation de la charge maximale $L_{m}$ à respecter en vue d'obtenir un certain stock dans le lac. Pour atteindre l'objectif de $[\mathrm{P}]=20 \mathrm{mg}^{-3}(\mathrm{P}=1800 \mathrm{t})$ préconisé par la CIPEL (1984, p. 572), les apports devraient se situer autour des $800 \mathrm{t}$, si l'on se base sur $\mathrm{f}_{\mathrm{h}}$, ou seulement de 350 $t$, en applicant $\pi$ ! La surestimation de la capacité éliminatrice du lac causée par l'utilisation de $f_{h}$ a pour conséquence la fixation d'objectifs trop " optimistes "dans la réduction des apports au lac.

\section{4. - Evolution chronologtque de $\varrho, \beta, \sigma$ et $\phi$}

On a vu (Tableau II) que nombre de paramètres du modèle varient fortement au cours du temps. En vue de pratiquer une simulation du système aussi réaliste que possible, il convient de déterminer si ces paramètres ont un comportement chronologique aléatoire, ou au contraire suivent une tendance. périodique ou non. Un test de " runs " (Miller et Kahn 1962, p. 325 sq) a été appliqué, sans approximation normale. Ses résultats sont reportés dans le Tableau IV.

Des quatre paramètres en question, $\varrho, \sigma$ et $\phi$ peuvent être considérés comme ayant un comportement chronologique alèatoire. Pour $\beta, \mathrm{P}(\mathrm{u})$ est légèrement au-dessous du seuil de 0,05 , indiquant une tendance à l'autocorrélation (Jaquet 1974), d'ailleurs visible
Tableau IV. - Tests de * runs s sur l'évolution de divers paramètres $(\mathrm{P}(\mathrm{u})$ : probabilité que la série soit aléa toire).

\begin{tabular}{|c|c|c|c|c|c|}
\hline Paramecre & "s & $n 1$ & $n z$ & $F(u)$ & Alotente \\
\hline 1 & 11 & 10 & 16 & 0,28 & out \\
\hline E & 5 & 10 & 10 & 0.014 & non \\
\hline$P$ & 10 & 11 & 9 & 0.18 & oul \\
\hline$B$ & 7 & is & 9 & 0.035 & non \\
\hline 0 & 12 & 10 & 10 & 0.18 & OU1 \\
\hline 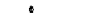 & 12 & 1 & 9 & 0,15 & ou: \\
\hline
\end{tabular}

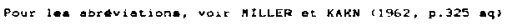

sur la fig. 4. Son caractere est clima tique (alternance d'hivers plus ou moins froids).

L'évolution temporelle du coefficient d'élimination $\phi$ pourrait donc êt re simulée pratiquement par une suite de valeurs tirées d'une distribution normale de moyenne $\phi$ et d'écart-type S $\phi$.

. Alors que la stationnarité de $\phi$ peut être établie sur la base du test de " runs ", il n'en va pas de même de $S \phi$ : la fig. 4 montre clairement qu'à partir de $1972, \phi$ varie moins autour de sa moyenne. Il faudra donc en tenir compte lors de la simulation.

\section{5. - Simulation}

\section{1. - Introduction}

Dans le cadre du modèle simple d'entrées-sorties, le but de la simulation est de prédire le stock de phosphore total dans le lac en fonction des apports et du coefficient d'élimination.

Classiquement, cette démarche est accomplie de deux manières différentes:

1. Le coefficient d'élimination $\phi$ est approximé par $\varrho$, en général selon la relation (11), en supposant que le système est à l'équilibre avec les apports et que $e$ est constant. On utilise ensuite les diagrammes empiriques de l'OCDE (1982) reliant [P]i à [P]. C'est l'approche qui a été utilisée par la CIPEL (1984, p. 569) pour le Léman et par Vollenweider et al. (1979) pour les Grands Lacs. Notons que dans ce dernier cas, l'application du modèle simple a donné des résultats analogues à ceux d'un modèle plus complexe ( 2 niveaux, 2 compartiments). Pour le Léman, on a vu plus haut que l'approximation de $\phi$ à l'aide de e n'était pas satisfaisante.

2. Le coefficient $\phi$ est soit mesuré, soit calculé à l'aide de la relation du Tableau I, en supposant que le système est à l'équilibre et que $\phi$ est constant. 
Comme ce dernier est très rarement mesuré (absence de données sur la sédimentation), on l'obtient par calcul. On applique ensuite des relations du type (12) si l'on veut simuler l'évolution du stock, ou (13), si l'on s'intéresse simplement au stock à l'équilibre (Sonzogni et al. 1976).

Dans le cas du Léman, la grande variabilité de $\phi$ au tour de sa moyenne limite beaucoup l'intérêt de cette approche. Une autre démarche a donc été ima. ginée, basée sur la simulation récursive du stock à l'aide de l'équation du bilan (6) réarrangée ainsi pour une ième année.

$$
\mathbf{P J}_{\mathbf{i}+\mathbf{1}}=\mathbf{L}_{\mathbf{i}}+\mathbf{P J}_{\mathbf{i}}-\phi \mathbf{P}_{\mathbf{i}}
$$

où $P J_{i}$ est le stock en janvier et $P_{i}$ le stock annuel moyen. Pour pouvoir utiliser (19), il faut poser PJ = P. Le bien-fondé de cette hypothèse simplificatrice a été testé sur les données 1964-1983, pour lesquelles on a remplacé $\mathbf{P}$ par $\mathbf{P J}$. Ces deux variables sont corrélées significativement $(r=0,93)$. Il en va de même entre $P J$ simulés et mesurés $(r=0,91)$. Il est donc justifié de poser :

$$
\mathbf{P J}_{\mathbf{i}+1}=\mathrm{L}_{\mathrm{i}}+\mathbf{P J}_{\mathrm{i}}(1-\phi)
$$

où $\phi$, à chaque pas est généré pseudoaléatoirement à partir d'une distribution normale de moyenne et variance données. L est gardé constant ou varie comme :

$$
\mathrm{L}_{\mathrm{i}}=\mathrm{L}_{\mathrm{i}-1 \mathrm{a}}
$$

\section{2. - Approche déterministe}

En dépit des critiques mentionnées ci-dessus, nous présentons quelques résultats de simulation déterministe de type 2 , afin de permettre la comparaison avec l'approche stochastique.

Les conditions sont celles des années 80 : stock de débart $8000 \mathrm{t}$ et $\mathrm{L}$ constant à $1200 \mathrm{t}$ an-1. On a simulé l'évolution du stock pendant 60 ans pour des valeurs de $\phi$ constantes dans le temps : $-0,001,0,05$, $0,15,0,20$ et 0,90 an $^{-1}$ (fig. 5 ). On a aussi reporté sur cette figure l'évolution observée de 1980 à 1983.

Selon les valeurs de $\phi$, le stock peut augmenter $(\phi>0,15)$, rester stationnaire ou diminuer sans que L varie. Dans le cas optimiste d'une bonne sédimentation $(\phi=0,25)$, le stock atteindrait un quasiéquilibre en 8-10 ans. Dans le cas très pessimiste d'un $\phi$ durablement inférieur à 0,15 , cet te équilibration prendrait plusieurs décades. Notons qu'avec cette charge et un $\phi$ réaliste, il est pratiquement exclu que le stock descende au-dessous de $5000 \mathrm{t}$.

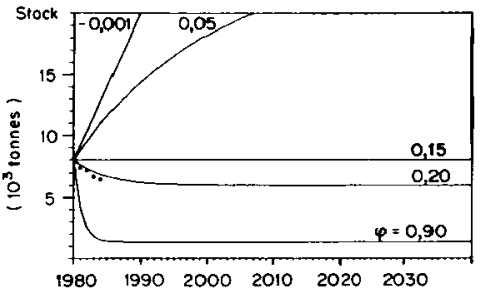

Fig. 5 : Scénarios d'évolution du stock de Ptot pour $\mathbf{L}=$ $1200 t^{-1} a^{-1}$ et diverses valeurs de $\phi$ maintenues constantes au cours du temps (simulation déterministe).

\section{3. - Approche stochastique}

\subsubsection{Principes}

La parfaite régularité des courbes de la fig. 5 provient de la constance de $\phi$ sur plusieurs décennies, ce qui n'est pas réaliste. On a vu que ce coefficient varie aléatoirement autour d'une moyenne. Cette dernière a été choisie à $0,20 \mathrm{an}^{-1}$, correspondant à la norme 1964-1983.

Pour ce qui est de la dispersion, les valeurs suivantes de $\mathbf{S} \phi$ ont été testées lors des simulations: $0,20,0,10,0,05$ et 0,01 , ce qui, pour $\phi$, représente un coefficient de variation de $100,50,25$ et $5 \%$ respectivement.

Les apports ont été soit gardés constants au niveau des années 80 , soit réduits de 20 ou $40 \mathrm{t}$ $a^{-1}$. Ces réductions conduiraient au respect des normes de la CIPEL $\left(\mathrm{L}=500 \mathrm{t}^{\mathrm{an}}{ }^{-1}\right)$ après un laps de temps de 35 et 17 ans respectivement.

Enfin, chacun de ces scénarios a été simulé selon trois réalisations de la série chronologique pseudoaléatoire de $\phi$. Les résultats reportés sur les fig. 6 à 8 représentent les valeurs du stock en janvier; les courbes du stock moyen auraient une allure analogue, mais légèrement plus émoussée.

\subsubsection{Apports constants}

Sur la fig. 6, les simulations placées sur la même ligne (même réalisation de $\phi$ ) montrent une variabilité autour de la courbe moyenne ( $S \phi=0)$ décroissant linéairement avec la valeur de $\mathbf{S} \phi$. Il s'agit maintenant de choisir la dispersion la plus vraisemblable. Pour ce faire, on a calculé la moyenne de l'écart absolu interannuel (|dPJ|) pour les données 

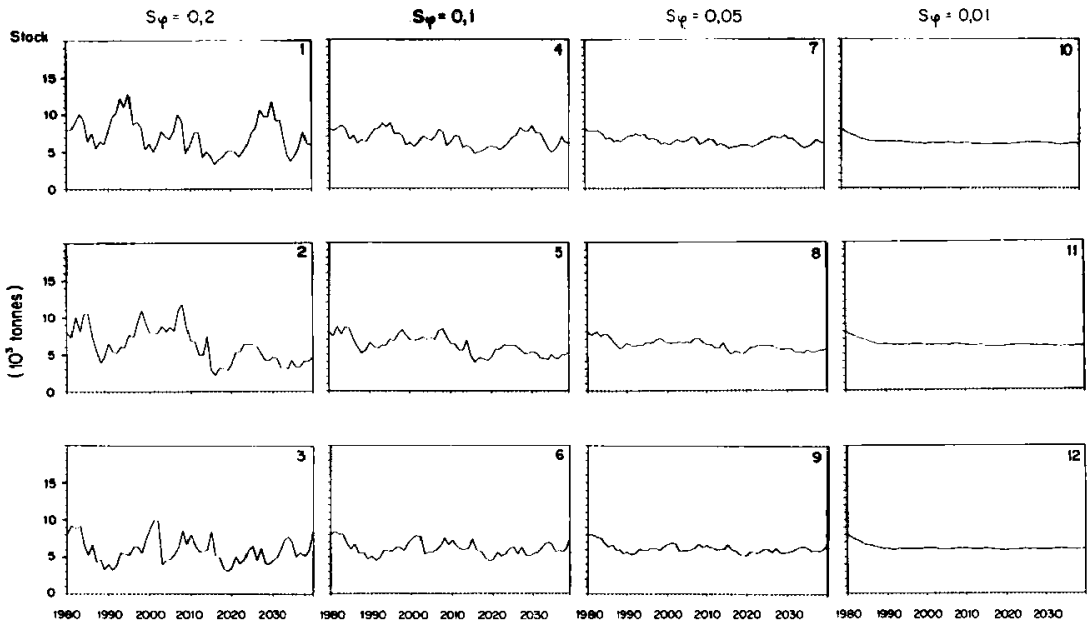

$1990 \quad 1990 \quad 2000 \quad 2010 \quad 2020 \quad 2030$

$1960 \quad 1990200020102020 \quad 2030$

199019902000201020202030

$1980 \quad 2000201020202030$

Fig. 6: Scénarios d'évolution du stock de Ptot pour $\mathrm{L}=1200 \mathrm{tan}^{-1}, \phi=0,2 \mathrm{an}^{-1}$ et diverses valeurs de $\mathrm{S} \phi$
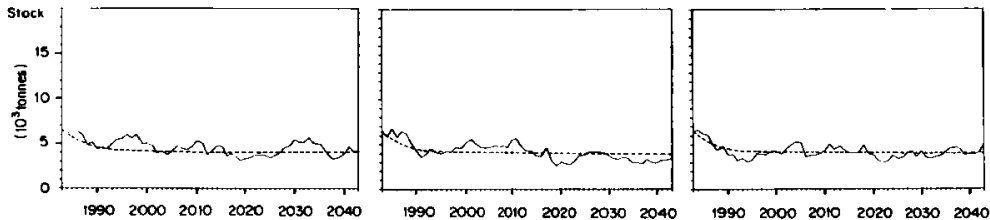

Fig. 7 : Evolution du stock de Ptot à partir de 1983 suite à une diminution intantanée des apports à $800 \mathrm{t} \mathrm{an}^{-1}$. 


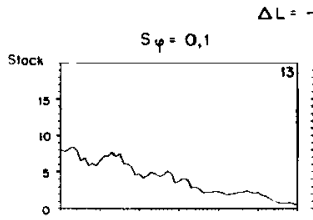

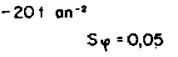
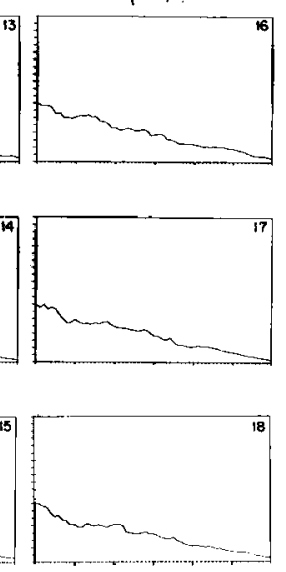

$\$ 9801900200020102020 \quad 2030$
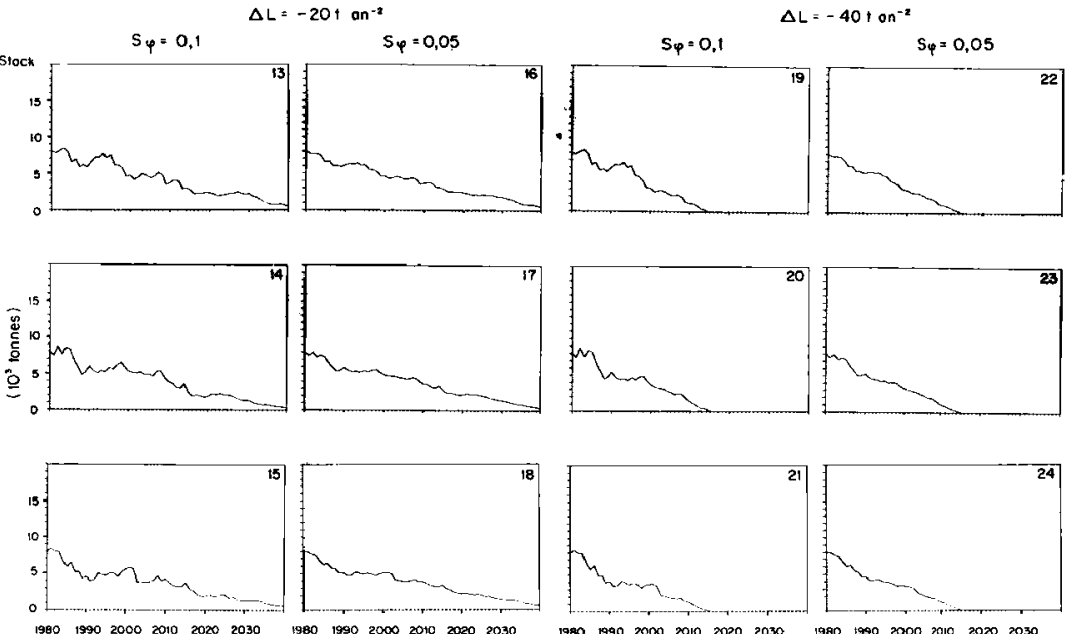

$1960 \quad 199020002010 \quad 2020 \quad 2030$

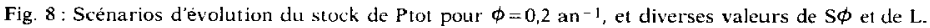

simulées et observées (Tableau $V$ ) ; ces dernières ont été prises de 1976 à 1983, période pour laquelle on peut considérer les apports comme à peu près conslants. IdPJ| vaut alors environ $600 \mathrm{t} \mathrm{an}^{-1}$. Cet ordre de grandeur de variabilité du stock est simulé par les cas 4-6 $\left(|\mathrm{dPJ}|_{\mathrm{s}}=560 \mathrm{t}^{-1} \mathrm{an}^{-1}\right.$, donc lors $\mathrm{S} \alpha=0,1$.

Cela signifie que l'évolution prévisible du stock en cas de constance des apports à $1200 \mathrm{t}_{\mathrm{an}^{-1}}$ et d'une variation réaliste de $\phi$ oscillera autourd'une valeur de $6000 t$, dans une fourchette de \pm 3000 tenviron. On doit donc s'attendre à d'importants renversements de tendance $(\mathrm{dP}>0)$ sur une durée de plusieurs années.

Un autre scénario intéressant (fig. 7) implique la réduction rapide des apports suite à une interdiction des phosphates dans les détergents (OFPE, 1983 ; scénario C, annexe p. 2). La charge tombera à $800 \mathrm{t} \mathrm{an}^{-1}$. A partir d'une valeur de $6500 \mathrm{t}$ en 1983, le stock évoluera vers un état d'équilibre à $4000 \mathrm{t}$, avec un $|\mathrm{dPJ}|_{\mathrm{s}}=390 \mathrm{t} \mathrm{an}^{-1}$. Là encore, des inversions de tendances pourraient faire remonter le stock jusqu'à 6000 t. D'autre part, une succession
Tableau V. - Valeur moyenne de l'écart absolu interannuel $|\mathrm{dPJ}|$ du stock de Ptotal pour les séries observées et simulées $(\mathrm{dL}=0)$.

\begin{tabular}{|c|c|c|}
\hline $\begin{array}{l}\text { No de la } \\
\text { simulation }\end{array}$ & $\sin ^{-1}$ & $\mid \begin{array}{l}\mid \mathrm{dPJ} \\
{[a n}\end{array}$ \\
\hline $1-3$ & 0.2 & 1130 \\
\hline $4-6$ & 0,1 & 250 \\
\hline $7-9$ & 0.85 & 200 \\
\hline $20-12$ & 0,02 & $\infty$ \\
\hline $\begin{array}{l}\text { Serle observee } \\
1976-83\end{array}$ & 0.12 & 610 \\
\hline
\end{tabular}

de valeurs relativement fortes de $\phi$ pourraient l'abaisser autour des $3000 \mathrm{t}$.

En aucun cas toutefois, des apports à $800 \mathrm{tan}^{-1}$ ne pourraient conduire au stock-cible de $2000 \mathrm{t}$. 


\subsubsection{Apports variables}

Dans l'hypothèse d'une dimimution régulière des apports, on a tout d'abord simulé l'évolution du stock pour $\mathrm{dL}=-20 \mathrm{et}-40 \mathrm{tan}^{-2}, \mathrm{~S} \phi=0,1$ et 0,05 ( $f$ i, 8 ). Ainsi, les cas 13-15 correspondent-ils aux cas 4-h de la fig. 6. On notera toutefois une différence importante entre les séries à $\mathrm{dL}=0$ et $\mathrm{dL}<0$ : ces dernières montrent une variabilité absolue diminuant avec la valeur de PJ. Cela est dû au fait que :

$$
|\mathrm{dPJ}|=\mathbf{S} \phi \mathbf{P J}
$$

Dans le cas de $\mathrm{dL}<0$, on peut aussi démontrer que, pour $\mathrm{t}>2,3 \tau \mathrm{p}$, la pente moyenne de la courbe du stock vaut :

$$
\mathrm{dPJ}=\mathrm{dL} / \boldsymbol{\phi}
$$

et que le temps pour arriver à une valeur désirée Pm du stock est :

$\mathbf{t}_{\mathrm{pm}}=\left[\mathrm{L} / \mathrm{dL}+\tau_{\mathrm{p}}\right]\left[1-\mathrm{Pm} / \mathrm{P}^{0}\right]$

Dans le cas d'une diminution de $20 \mathrm{t} \mathrm{an}^{-1}$, il faudra presque 50 ans pour atteindre la valeur de 2000 t recommandée par la CIPEL (1984). En outre, $|\mathrm{dPJ}|$ passera d'environ $600 \mathrm{t}$ les premières années à $200 \mathrm{t}$ au moment d'atteindre ce seuil, et les inversions de tendance iront en diminuant.

\section{6. - Conclusions}

Le bilan d'entrées-sorties du phosphore total, calculé pour une vingtaine d'années, révèle une succes. sion assez chaotique d'épisodes à sédimentation ou réoénération nettes. La prédominance de l'un ou de l'autre de ces processus dépend essentiellement des conditions météorologiques par le biais des mélan. ges hivernaux incomplets (désoxygénation des fonds) et des apports particulaires, favorisant la sédimentation. Ce bilan a pu être vérifié en 1980-81, grâce à des mesures de sédimentation, qui ont confirmé les valeurs calculées $(S=1200 \mathrm{t}$ ).

L'expression de ce bilan à l'aide d'un modèle simple du type Vollenweider est possible, moyennant les hypothèses habituelles. Celle de linéarité peut être acceptée, mais seulement si l'on considère la moyenne pluriannuelle du coefficient d'élimination $\phi$. La grande variabilité de ce dernier provient en partie de la mauvaise estimation des apports externes avant 1970, mais aussi de la variabilité intrinsèque du coefficient de sédimentation $\sigma$. Il est urgent d'at taquer l'étude détaillée de ce dernier processus, qui domine largement dans l'élimination du phosphore hors du système. Il en va de même de la biodisponibilité des apports en Ptotal, dont l'élucidation permettra de déboucher sur une véritable modélisation de l'état trophique et biologique du lac (Burrus 1983).

Une autre caractéristique importante du cycle lémanique du phosphore est le déséquilibre existant, après 1958, entre apports et stock. Les variations de la charge ont été suffisamment rapides par rapport au temps de réaction du lac (une vingtaine d'années) pour que le stock mesuré soit inférieur au stock à l'équilibre jusqu'en 1980, et supérieur depuis lors.

Ce déséquilibre explique en partie le comportement " marginal " du Léman constaté par la CIPEL (1984). Il doî aussi inciter à la prudence dans l'application des normes OCDE (1982), particulièrement lors de l'approximation du coefficient d'élimination par celui de renouvellement de l'eau (e).

Enfin, variabilité de $\phi$ et tendance au déséquilibre rendent difficile la modélisation du cycle du phosphore à l'aide des équations classiques, telles que (12) ou (13). Pour tenir compte de ces conditions, il est avantageux de recourir à une simulation de type stochastique. La valeur moyenne choisie pour $\phi\left(0,20 \mathrm{an}^{-1}\right)$ et sa dispesion $(0,10)$ peuvent être considérées comme réalistes sur la base des années récentes. Nous n'avons pas postulé de tendance pour cette valeur moyenne, bien que l'on puisse imaginer une meilleure élimination suite à l'amélioration du niveau trophique (Vollenweider 1983). Le mérite principal de cette approche est la mise en évidence, pour les scénarios à charge constante, de fluctuations interannuelles du stock pouvant atteindre quelques milliers de tonnes et d'inversians de tendance pouvant s'étendre sur plusieurs années (fig. 6).

On démontre aussi qu'au niveau actuel des apports $\left(1200 \mathrm{t}^{-1}\right)$, il est impossible d'at teindre les objectifs de stock fixés par la CIPEL (2000. $3000 \mathrm{t}$ ), à moins d'admettre une valeur totalement irréaliste du coefficient $\phi$. Il en va de même dans l'éventualité d'une interdiction totale de phosphates dans les détergents, à la suite de laquelle le stock se stabiliserait autour des $4000 \mathrm{t}$.

Si l'on postule enfin une diminution des apports de $20 \mathrm{tan}^{-2}$, il faudra patienter une cinquantaine d'années avant d'arriver à l'objectif des 2000 t. Au début de cette phase de catatrophie provoquée, il y aura également des inversions de tendance, des " rechutes " graves dues à la variabilité incontrôlable 
du coefficient d'élimination. C'est alors qu'il faudra faire preuve de tenacité dans les efforts pour diminuer les apports externes. Dans un lac de la taille du Léman, la manipulation de $\phi$ est pour le moment utopique, et la seule stratégie réaliste de contrôle du stock passe par la limitation des apports externes.

\section{Remerciements}

Ce travail représente la première étape d'un projet de compréhension multidisciplinaire de l'écosystème lémaninue (MODEC) lancé à l'Institut de Limnologie de Thonon. Nous sommes reconnaissants à J.-B. Lachavanne et aux autres chercheurs de l'UBA pour la part qu'ils ont prise darss la maturation de nos idées sur le cycle du phosphore dans le Léman.

\section{Travaux cités}

Ammann (P.). 1984 - Les bilans de phosphore au Léman. Présentàtion Journée Technique ARPEA (Responsabilité de L'agriculture à l'eut rophisation des lacs). Ecole Polytechnique Fédérale de Lausanne, Institut de Génie Biologique, $13 \mathrm{p}$.

Burrus (D.). 1984. - Contribution à l'élude du transport du phosphore dans le Rhóne alpin. Thèse Université de Genève, $\pi^{\circ}$ $2135,100 \mathrm{p}$.

CIPEL (Commission Internationale pour la Protection des Eaux du Lejman contre la Pollution). 1984: Rapport de synthèse 1964.1980, 647 p., Lausanne (Suis se).

Dillon (P.J.). 1974. - A critical review of Vollenweider's nutrient budget model and other related models. Water Resources Bull., Am. Water Res. Assoc., 10 (5) : 969.989.

Edmondson (W.T.) et Lehman (J.T.). 1981. - The effect of changes in the nutrient income on the condition of Lake Washing. ton. Limnol. Oceano., 26 (1): 1-29.
Gaechter (R.). Imboden (D.) et Joller (T.). 1983, - Lake Restora(ion. In - Extended Abstracts of the workshop on chemical pro cesses in lakes : : 119.127. EAWAG, Dübendorf, Suisse.

Haubert (M.), Siwertz (E.), Chassaing (B.) et Olive (P.). 1975. Apports en nutrients au lac Lérnan pour la période 1963-1972. Arch. Sciences Genève, 28 (1): 41-52.

Jaquet (J.-M.). 1974. - Analyse statistique de la variation verticale des descripteurs dans la Formation de Vions. A rch. Sciences Genève, 26 (3) : 247-283.

Jaquet (J.-M.). 1979. - Formes du phosphore dans les sédiments lémaniques en 1978. Rapport CIPEL 1978 : 310-326.

Jaquet (J.-M.), Favarger (P.-Y.), Uldry (A.) et Vernet (J.P.). 1983. - Premières données sur la matière particulaire dans le Leman. Rapport interne Institut Forel, Genève, 83 p.

Jaquet (J.M.) et Snodgrass (W.J.). 1984. - Modélisation du phosphore. In * Rapport de synthèse *: 549-558 CIPEL, Lausanne.

Lachavanne (J.-B.). 1980. - Les manifestations de l'eutrophisation d'un grand lac profond: le Léman (Suisse). Revue Suisse Hydrol, 42 (2) : 127.154.

Miller (R.L.) et Kahn (J.S.). 1962. - Statistical analysis in the geological sciences. Wiley. New York, 483 p.

OCDE, 1982. - Eutrophisation des Eaux : méthodes de surveil. lance, d'évaluation et de lut te. Organisation de coopération et de développement économiques, Paris, $164 \mathrm{p}$.

OFPE (Office Fédéral de la Protection de l'Environnement), 1983 : - Phosphates dans les lessives. Cahiers de l'Environnment, no 14, 68 p., Berne.

Premazxi (G.) et Rossi (G.). 1984. - Phosphorus cycle in a eutrophic subalpine lake. Final report. Commission des Communau. lés européenes, Centre de Jecherches d'Ispra, $56 \mathrm{p}$.

Sonzogni (W.C.), Uttormark (P.C.) et Lee (G.F.). 1976. - A phosphorus residence time model : theory and applications. Water Research, $10: \mathbf{4 2 9 - 4 3 5 .}$

Vollenweider (R.). 1968. - Scientific Fundamentals of the Eutrophication of Lakes and Flowing Waters, with particular Reference to Nitrogen and Phosphorus as Factors in Eutrophica. tion. Tech, Report DAS/SCL68.27, OECD, Paris, 159 p.

Vollenweider (R.), Rast (W.) \& Kerekes (J.). 1979. - The phosphorus loading concept and Great Lakes eutrophication. In $\propto$ Phosphorus Management Strategies for Lakes $x$, chap, 9: 207-233.

Vollenweider (R.). 1983, - Notion de charge admissible. Commu. nication présentée au Colloque surl'eutrophisation et la polltution du Léman, org. ASL-CIPEL, Genève, sept. 1983, 11 p. 